See discussions, stats, and author profiles for this publication at: https://www.researchgate.net/publication/309828045

\title{
Clinical Comparison of Two Photosensitizers for Oral Cavity Decontamination
}

Article in Photomedicine and laser surgery · November 2016

DOI: 10.1089/pho.2016.4114

CITATIONS

6 authors, including:

Sebastião Pratavieira

University of São Paulo

90 PUBLICATIONS 450 CITATIONS

SEE PROFILE

Aldo Brugnera Junior

118 PUBLICATIONS 1,548 CITATIONS

SEE PROFILE

Some of the authors of this publication are also working on these related projects:

Project laser bracket debonding View project

Project Effects of Low Level Laser in the compensatory hypertrophy View project
READS

62

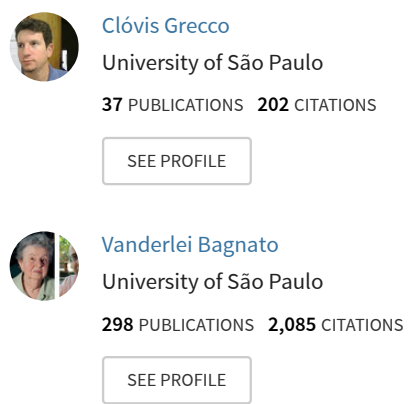




\title{
Clinical Comparison of Two Photosensitizers for Oral Cavity Decontamination
}

\author{
Hérica Adad Ricci Donato, PhD, ${ }^{1,2}$ Sebastião Pratavieira, $\mathrm{PhD}^{2}$, Clovis Grecco, PhD, \\ Aldo Brugnera-Júnior, $\mathrm{PhD}^{3}$, Vanderlei Salvador Bagnato, $\mathrm{PhD}^{2}$, and Cristina Kurachi, $\mathrm{PhD}^{2}$
}

\begin{abstract}
Objective: In this study, we aim to compare the photodynamic inactivation (PDI) effects of two different photosensitizers (PS), Photogem ${ }^{\circledR}$ and Natural Curcumin, irradiated with light-emitted diodes (LED) at 630 and $450 \mathrm{~nm}$, respectively. Background: The current antimicrobial mouthwash for oral hygiene has several drawbacks. In this context, PDI is an alternative technique to inactivate pathogenic microbes in mucosa and in periodontal tissue. Furthermore, there are numerous infectious diseases that may affect the oral cavity, motivating the use of PDI in dentistry. Methods: The volunteers $(n=50)$ were randomize separated into five experimental groups $(n=5)$ for each PS: water control, PS control, light control, and two PS concentrations (25 and $100 \mathrm{mg} / \mathrm{L}$ ). Each patient underwent mouthwash solution containing the PS before illumination procedure that was performed with an LED device. For microbial decontamination evaluation, the saliva was collected three times: before (T0), immediately after (T1), and $24 \mathrm{~h}$ after the illumination procedure (T2). After that, the difference between the colony forming units (CFU) for each volunteer was compared. Results: The results show that regardless of PS and treatment applied, there was microbial reduction immediately after PDI, however, after $24 \mathrm{~h}$ only Natural Curcumin still presents a reduction. For Photogem after $24 \mathrm{~h}$, the microorganism returns to the original CFU. Conclusions: Immediately after PDI, both PS have the same efficiency, nevertheless the Natural Curcumin still has an efficacy after $24 \mathrm{~h}$ and also is a more viable photosensitizer. In addition, the results indicate that PDI can be a promised technique used for microbial reducing for the oral cavity.
\end{abstract}

Keywords: photodynamic inactivation, in vivo, clinical, curcuminoids, hematoporphyrin

\section{Introduction}

$\mathbf{T}$ HE ORAL CAVITY is a favorable environment for the growth of various microorganisms due to its conditions such as humidity, temperature, and abundant presence of food waste. It is estimated that more than 700 species of microbes inhabit the human oral cavity, in which bacteria are predominant. ${ }^{1}$ In this sense, antibiotics have become routinely used in the dental office, especially in the control of postoperative infections after oral surgeries. The antibiotic therapy has provided in the field of infectious disease control the most revolutionary change throughout the history of medicine. However, microbial resistance due to the abusive and nonjudicious use of bacterial agents is now considered a global problem. ${ }^{2}$

Such resistance to current antibiotics has limited the cure of diseases, leading to a growing interest in alternative therapies to combat pathogenic microorganisms. The most frequent infectious diseases that affect the oral cavity are periodontal disease, caries, endodontic infections, and oral candidiasis associated with the use of oral prostheses. A first alternative approach is the use of local methods such as the use of antimicrobial mouthwash that has been proposed as a means of reducing the levels of oral bacteria. In this sense, the chlorhexidine has been widely used in various clinical situations in dentistry. ${ }^{3}$ Chlorhexidine has a broad spectrum of action, acting on gram-positive bacteria, gram-negative bacteria, fungi, lipophilic yeasts, and viruses. The action of chlorhexidine mouthwash persists after more than $7 \mathrm{~h}$ with bacterial reduction $\geq 90 \%$. Thus, it exerts a bactericidal action that starts immediately after the mouthwash, combined with a sustained bacteriostatic effect, which prevents bacterial colonization and residual inhibitor effect of the formation of dental plaque. ${ }^{4}$ Nevertheless, some side effects are attributed to prolonged oral use, for more than 14 days, such as brownish stains on the teeth, restorations, or in the

\footnotetext{
${ }^{1}$ School of Dentistry of Araraquara, UNESP_University of Estadual Paulista, Araraquara, Brazil.

${ }^{2}$ São Carlos Institute of Physics, University of São Paulo, São Carlos, Brazil.

${ }^{3}$ Biomedical Engineer Research Center, CEB, Camilo Castelo Branco University, São Jose dos Campos, Brazil.
} 
back of the tongue, peeling and loss of oral sensitivity, bitter taste, and interference with taste sensation. Among these effects, the dental staining stands out as the main complaint from patients, the main factor limiting the use of chlorhexidine for prolonged periods. ${ }^{5}$

In this context, photodynamic inactivation (PDI), antimicrobial photodynamic therapy (aPDT), or photodynamic antimicrobial chemotherapy (PACT) has arisen as a promising alternative to microbial control. ${ }^{6,7}$ The photodynamic reaction is induced by the interaction of a photosensitizing agent (PS) with light in the presence of molecular oxygen. The PS accumulates selectively in cells or microorganisms or binds to its outer surface (membrane or cell wall) and is then carried the irradiation of the target tissue with light at a suitable wavelength. ${ }^{7-9}$ The microbial reduction by PDI has been shown effective in various organisms, from fungi, protozoa, viruses to even undesirable bacteria. ${ }^{10-12}$

In dentistry, this treatment has been proven highly effective in endodontic treatments, stomatitis by Candida albicans, ${ }^{6,13,14}$ and periodontics. ${ }^{15}$ For conditions, such clinical situations, which require prior microbial control, patients undergo various forms of oral surgery to prevent contamination of the surgical site and thus obtain adequate tissue repair. ${ }^{16}$ PDI is also convenient instead of mouthwashes such as chlorhexidine, which has proved itself ineffective in preventing the establishment of a potentially pathogenic microbiota. ${ }^{17}$

The present study compares the in vivo PDI effects of two different photosensitizers (PS) as mouthwashes for reducing microorganisms in the oral cavity. The PS used were Photogem and curcuminoids.

Photogem $^{\circledR}$, a hematoporphyrin derivative, is widely used as PS in cancerous lesion treatment with Photodynamic Therapy (PDT). ${ }^{18}$ It also had good answers in the inactivation of some microorganisms ${ }^{19,20}$ and has been tested, for example, on Streptococcus mutans and Lactobacillus acidophilus of carious dentin, ${ }^{20}$ Candida albicans and Candida glabrata resistant to fluconazole, ${ }^{21}$ and Staphylococcus aureus. ${ }^{22}$ All these microorganisms can be found in the oral microbiota.

The main curcuminoids are curcumin, demethoxycurcumin, and bisdemethoxycurcumin. Curcuminoids are yellow pigments found in the rhizome of turmeric (Curcuma longa L.). ${ }^{23}$ Many effects have been reported for curcumin, including immunomodulatory, anti-inflammatory, antioxidant, and antitumor effect. ${ }^{24-26}$ Curcuminoids also have antibacterial effects and have been used as PS in PDI to treat infections, ${ }^{27}$ in the inactivation of methicillin-resistant strains of Staphylococcus aureus, ${ }^{28}$ in species of Candida albicans, ${ }^{13,29}$ in Gram-negative and Gram-positive bacteria, ${ }^{27,30}$ and in larvae of mosquitoes. ${ }^{31}$ Two similar clinical trials were already performed with curcuminoids, one used a salt form of curcumin and the other just explores one curcumin concentration. ${ }^{32,33}$

\section{Material and Methods}

\section{Selection of volunteers}

In total, 50 healthy volunteers of both genders aged 18-40 years, who signed written informed consent, previously approved by the Ethics Committee in Research (1376182012-Federal University of São Carlos), were enrolled in the study. The volunteers were randomize separated into two main groups with five experimental conditions $(n=5)$ for each PS: water control, PS control, light control, and two PS concentrations ( 25 and $100 \mathrm{mg} / \mathrm{L}$ ). This sample size was also based on previous studies. ${ }^{32,33}$ The exclusion criteria of patients in this study were patients with uncontrolled systemic diseases, those who underwent treatment with systemic antibiotic therapy in the last 6 months, those who make continuous use of some type of mouthwash, and smokers.

\section{Photosensitizers}

For this study, the following two photosensitizers, Photogem (Photogem Limited Liability Company, Moscow, Russia) and Natural Curcumin (PDT Pharma, Cravinhos, Brazil), were used.

Natural Curcumin is a mixture of curcuminoid pigments (60\% curcumin and $40 \%$ bisdemethoxycurcumin, demethoxycurcumin) and was first solubilized in $99.9 \%$ of absolute ethanol and $0.1 \%$ of dimethyl sulfoxide-DMSO. From a stock solution at $0.15 \%$, final solutions were obtained for testing with 25 and $100 \mathrm{mg} / \mathrm{L}$ in distilled water. Photogem was also tested at two concentrations, 25 and $100 \mu \mathrm{g} / \mathrm{mL}$; to obtain such final concentrations, only distilled water was added to the stock solution of $5 \mathrm{mg} / \mathrm{mL}$.

As for the control groups, in addition to the water control group, in which patients received only a mouthwash with distilled water (LOCO and LOP0), there was also a photosensitizer-only control group, which underwent three types of mouthwash with curcumin $100 \mathrm{mg} / \mathrm{L}$ (L0C100) or Photogem (L0P100), and no illumination of the oral cavity. These PS concentrations were based on the literature. ${ }^{32,33}$

Before application of the light source, the patient underwent three mouthwashes for $1 \mathrm{~min}$ each, with $15 \mathrm{~mL}$ of a solution containing one of the PS of choice, always monitored by a professional and without swallowing the solution.

\section{Irradiation}

For irradiation, immediately after mouthwash, two homemade light-emitted diode (LED) devices were used as light sources. A device emitting in the range of blue light at $450 \mathrm{~nm}$ for Natural Curcumin and the other emitting in the range of red light at $630 \mathrm{~nm}$ for Photogem, with a light intensity of $\sim 100 \mathrm{~W} / \mathrm{cm}^{2}$. The diffuser tip of both LED apparatus was placed in the patient's mouth in a central position for $6 \mathrm{~min}$, the lips remained sealed for no dispersion of light during illumination. A control group with light only was also observed, a water mouthwash followed by the illumination with the $450 \mathrm{~nm}$ LED (COL6) or $630 \mathrm{~nm}$ LED (P0L6) depending on the photosensitizer tested. In Table 1, the studied groups are presented.

\section{Saliva samples and microbiological analyses}

For each patient, before the irradiation procedure (T0), saliva was collected using a falcon-type tube (labeled with name, date, and time of collection) where the patient spat up to a minimum of $1.0 \mathrm{~mL}$ of saliva. After irradiation, new saliva collection was made for counting of colony-forming unit (CFU) in the same way as described in the same protocol above. The salivary collection after lighting was held in two stages, immediately after irradiation (T1) and $24 \mathrm{~h}$ postirradiation (T2). 
Table 1. Control and Experimental Groups

\begin{tabular}{lll}
\hline PS & \multicolumn{1}{c}{ Groups } & \multicolumn{1}{c}{ Description } \\
\hline Natural Curcumin & COL0 $(n=5)$ & Absence of light and curcumin-water mouthwash \\
& C100L0 $(n=5)$ & Natural Curcumin mouthwash $100 \mathrm{mg} / \mathrm{mL}$ and absence of light \\
COL6 $(n=5)$ & Water mouthwash and illumination of $6 \mathrm{~min}$ \\
& C25L6 $(n=5)$ & Natural Curcumin mouthwash $25 \mathrm{mg} / \mathrm{mL}$ and illumination of $6 \mathrm{~min}$ \\
C100L6 $(n=5)$ & Natural Curcumin mouthwash $100 \mathrm{mg} / \mathrm{mL}$ and illumination of $6 \mathrm{~min}$ \\
Photogem & PoL0 $(n=5)$ & Pbsence of light and Photogem-water mouthwash \\
& P100L0 $(n=5)$ & Water mouthwash and illumination of 6 min \\
P0L6 $(n=5)$ & Photogem mouthwash $25 \mu \mathrm{g} / \mathrm{mL}$ and illumination of $6 \mathrm{~min}$ \\
P25L6 $(n=5)$ & Photogem mouthwash $100 \mu \mathrm{g} / \mathrm{mL}$ and illumination of 6 min \\
\hline
\end{tabular}

After being collected, samples were taken to the microbiology laboratory, where they suffered six successive dilutions in previously sterile saline and then were seeded in triplicate in droplets of $25 \mu \mathrm{L}$ on brain-heart infusion plates, a medium capable of supporting the growth of a wide range of microorganisms. The process of serial dilutions aims to reduce the concentration of CFUs, making the counting possible. Then, the plates were incubated at $37^{\circ} \mathrm{C}$ under aerobic conditions for about $48 \mathrm{~h}$. After the incubation period, counting of $\mathrm{CFU} / \mathrm{mL}$ was performed; this protocol was in accordance with similar previous studies. ${ }^{32-34}$

\section{Results and Discussion}

The counting of microorganisms- $\mathrm{CFU} / \mathrm{mL}$ of the oral cavity were expressing as the logarithm $\left(\log _{10}\right)$. The data are presented as changes in microbial reduction to two evaluation times: immediately after PDI (T1-T0) and $24 \mathrm{~h}$ after PDI (T2-T0). The results are shown in Fig. 1 for Natural Curcumin.

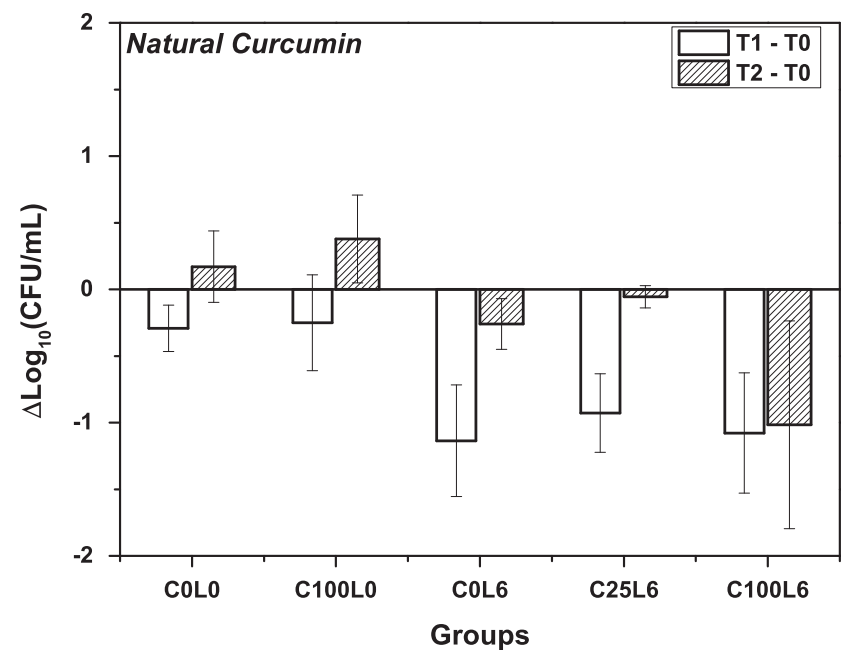

FIG. 1. Delta of reduction (T1-T0 and T2-T0) of colonyforming unities (log scale, Y-axis) of the oral cavity immediately after PDI (white box) and $24 \mathrm{~h}$ after (gray box) for five groups studied and their respective averages for Natural Curcumin. The results are plotted as columns, and the errors are presenting the standard error of the mean. PDI, photodynamic inactivation.
The analysis of the difference of microbial reduction was the choice for processing of experimental data of microbial count in different individuals with different microbial flora, allowing a comparison between patients of the same group.

After the treatment, microbial reduction was observed for all groups, even in the control group.

The average values show that post-PDI (T1-T0) occurs microbial reducing. Although the groups C0L6, C30L6, and C100L6 have promoted a high reduction rate, the control group had the greatest reduction rate. After $24 \mathrm{~h}$, the postPDI C100L6 group does not show microbial regrowth. On the contrary, remaining groups that show immediate reduction showed microbial regrowth $24 \mathrm{~h}$ after treatment and control curcumin (C100L0) showed the highest regrowth.

In Fig. 2 is presented the PDI for Photogem. According to the average values of reduction, it was observed that microbial reduction occurred immediately after PDI for all groups and P100L6 group shows the greatest reduction. However, $24 \mathrm{~h}$ after PDI, all groups showed a high rate of regrowth.

Decontamination of oral cavity becomes a relevant issue in situations ranging from routine before dental procedures

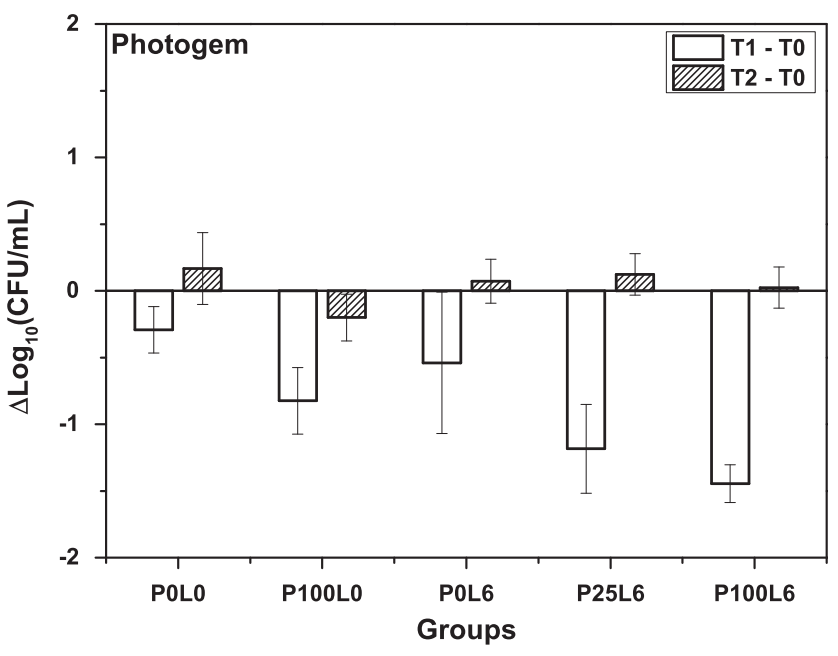

FIG. 2. Delta of reduction (T1-T0 and T2-T0) of colonyforming unities (log scale, Y-axis) of the oral cavity immediately after PDI (white box) and $24 \mathrm{~h}$ after (gray box) for five groups studied and their respective averages for Photogem. The results are plotted as columns, and the errors are presenting the standard error of the mean. 
to oral surgery. Therefore, systemic diseases have their origin in the oral cavity, which is considered the gateway to a large number of microorganisms.

The actuality of the topic justifies the variety of protocols used in this study. The concentration and the PS used, the irradiation exposure, and mouthwash time were variable parameters used because it is a new method for oral decontamination and has little support in literature..$^{32,33}$

The results show that Natural Curcumin was more effective in reducing oral microflora when was used at a higher concentration $(100 \mathrm{mg} / \mathrm{mL})$. Dovigo et al., ${ }^{34}$ in a study in vivo (with mice) using curcumin for performing PDI in the treatment of oral candidiasis, showed a significant reduction in the microbial concentration of $30 \mathrm{mg} / \mathrm{L}$. However, the brand used by the authors is different from that used in the present study, so that the purity of the product is not the same, thus justifying the concentration of curcumin required for efficiency thereof. The curcumin used by Dovigo et al. came from Sigma-Aldrich with $70 \%$ of purity, and the one used in our study is produced by PDT Pharma with $53 \%$ of purity.

In contrast, the Photogem had the highest average microbial reduction immediately after therapy when used at a concentration of $100 \mu \mathrm{g} / \mathrm{mL}$ followed by the concentration of $25 \mu \mathrm{g} / \mathrm{mL}$. Nevertheless, in all groups, this PS maintains the microbial reduction after $24 \mathrm{~h}$ of PDI, an unfavorable factor when the goal of oral intervention targeting oral decontamination over a longer term, such as in certain cases such as surgical tooth extraction and dental implant in periodontal scaling procedures such as among others.

For Photogem, the experimental group of $100 \mu \mathrm{g} / \mathrm{mL}$ (P100L6) had the highest microbial, although only immediate reduction (T0-T1). The choice of this concentration for our study was based on the antimicrobial effect of PDI used for surface disinfection of complete dentures in vivo study, where the concentration of $100 \mu \mathrm{g} / \mathrm{mL}$ was more effective, ${ }^{35}$ while the Photogem concentration of $25 \mu \mathrm{g} / \mathrm{mL}$ tested was supported by in vitro studies ${ }^{22}$ in which this concentration of PS associated with the light wavelength $628 \mathrm{~nm}$ was effective in microbial inactivation.

The control group only light (COL6) at a wavelength of $450 \mathrm{~nm}$ has shown high reduction in bacterial counts immediately after its application in the oral cavity, supported in the literature in which studies have shown the potential of blue light to promote bactericidal effect on different microorganisms such as $S$. aureus, E. coli, and $P$. gingivalis. ${ }^{36,37}$ However, the red light (POL6) alone did not show such great amounts of microbial reduction, which was also according to literature in which the irradiation wavelength at $625 \mathrm{~nm}$ was not able to exert a bactericidal effect on microorganisms previously cited. ${ }^{37}$

Although the observation of mean microbial reduction has allowed the previous statements, the lack of statistical significance between the results, independence PS tested, can be justified by several factors among own oral anatomy plus the type of lighting performed in the study. The lighting was central type, in which the diffuser tip was kept at rest supported on the tongue and in contact with the palate. In this position, the oral anatomy is very difficult to achieve light palatine and vestibular surfaces simultaneously, which detracted from the excitement of the PS and hence the effectiveness of PDI in these areas considered shade.
Regarding symptoms, some pertinent to curcumin group patients, independent of the concentration of PS or lighting time, reported pruritus during illumination after mouthwash, but always mild. The same symptoms were observed in the in vivo study of oral decontamination using curcumin and blue LED. ${ }^{33}$ As for the patients belonging to the group Photogem, no symptoms were reported. So far, for all individuals studied, any pain sensation and late effects such as the emergence of ulcers have been reported.

An important factor to be considered when dealing with oral decontamination is to change the color of teeth as well as aesthetic materials since the completion of the mouthwash is part of the protocol. Some substances such as toluidine blue O (TBO), a PS used effectively in PDI, ${ }^{20,38}$ can cause changes in the color of the tooth surface and resinous restorations due to their nature highly of pigmentation, ${ }^{20,39}$ which its contraindicate use in mouthwashes. However, the staining observed in the bovine enamel surfaces and composites using Photogem and curcumin is considered dependent on the storage time and the concentration, ${ }^{40}$ so the concentrations of PS used in this study and the short time of mouthwash present no potential for staining of teeth and/or restorations.

Among the PS used in this study, a factor that prevents the use of Photogem for oral decontamination besides not having submitted late effect of microbial reduction in the reported period $(24 \mathrm{~h})$ is the costliness of the product. Photogem is a hematoporphyrin derivative, and the cost of Photogem is about four times greater than curcumin; since this microbial reduction with PS was not proportionally increased, the cost effectiveness of this product, in this case, becomes impractical.

While many in vitro studies demonstrated the efficacy of PDI in reducing oral bacteria with different PS as TBO, ${ }^{38}$ Photogem, ${ }^{20}$ and curcumin, ${ }^{41}$ in vivo studies focused on oral decontamination become necessary to adjust the proposed protocols.

When compared with other therapies, PDI offers several advantages such as high target specificity, that is, the death of the bacteria can be controlled by restricting the irradiated region, besides few adverse side effects when using proper protocols, and yet no development of resistance by microorganisms, with viable cost due to the use of PS and inexpensive light sources. ${ }^{12,16}$ However, more studies need to be done to improve the microorganism reduction and make this an efficient clinical procedure.

\section{Conclusions}

Photodynamic reactions using Photogem and Natural Curcumin followed by a specific illumination are a promising technique for reduction of microorganisms in the oral cavity. Furthermore, from the average, we can conclude that Natural Curcumin at a concentration of $100 \mathrm{mg} / \mathrm{mL}$ with 6 min illumination has greater prominence by presenting microbial reduction immediately after PDI and maintenance of this reduction even after $24 \mathrm{~h}$ of therapy elapsed.

The use of photosensitizers can be a promised technique used for microbial reducing of the oral cavity. Because photosensitizers can be used in an ecofriendly approach, there is a lack of bacterial resistance induced in microorganisms. 


\section{Acknowledgments}

The authors acknowledge the support provided by Brazilian Funding Agencies: Capes; CNPq; and São Paulo Research Foundation (FAPESP) grant: 2013/07276-1 (CEPOF).

\section{Author Disclosure Statement}

No competing financial interests exist.

\section{References}

1. Perea EJ. Oral flora in the age of molecular biology. Med Oral Patol Oral Cir Bucal 2004;9 Suppl:6-10.

2. van Winkelhoff AJ, Winkel EG. Antibiotics in periodontics: right or wrong? J Periodontol 2009;80:1555-1558.

3. Matthijs S, Adriaens PA. Chlorhexidine varnishes: a review. J Clin Periodontol 2002;29:1-8.

4. Tomás I, García-Caballero L, Cousido MC, Limeres J, Alvarez M, Diz P. Evaluation of chlorhexidine substantivity on salivary flora by epifluorescence microscopy. Oral Dis 2009; 15:428-433.

5. Pratten J, Barnett P, Wilson M. Composition and susceptibility to chlorhexidine of multispecies biofilms of oral bacteria. Appl Environ Microbiol 1998;64:3515-3519.

6. Mima EG, Pavarina AC, Dovigo LN, et al. Susceptibility of Candida albicans to photodynamic therapy in a murine model of oral candidosis. Oral Surg Oral Med Oral Pathol Oral Radiol Endod 2010;109:392-401.

7. Hamblin MR, Hasan T. Photodynamic therapy: a new antimicrobial approach to infectious disease? Photochem Photobiol Sci 2004;3:436-450.

8. Allison RR, Bagnato VS, Cuenca R, Downie GH, Sibata $\mathrm{CH}$. The future of photodynamic therapy in oncology. Future Oncol 2006;2:53-71.

9. Ferreira J, Kurachi C, Moriyama LT, et al. Correlation between the photostability and photodynamic efficacy for different photosensitizers. Laser Phys Lett 2006;3:91-95.

10. Mang TS, Tayal DP, Baier R. Photodynamic therapy as an alternative treatment for disinfection of bacteria in oral biofilms. Lasers Surg Med 2012;44:588-596.

11. Jori G, Fabris C, Soncin M, et al. Photodynamic therapy in the treatment of microbial infections: basic principles and perspective applications. Lasers Surg Med 2006;38:468-481.

12. Donnelly RF, McCarron PA, Tunney MM. Antifungal photodynamic therapy. Microbiol Res 2008;163:1-12.

13. Dovigo LN, Pavarina AC, Ribeiro APD, et al. Investigation of the photodynamic effects of curcumin against Candida albicans. Photochem Photobiol 2011;87:895-903.

14. Mima EGDO, Pavarina AC, Silva MM, et al. Denture stomatitis treated with photodynamic therapy: five cases. Oral Surg Oral Med Oral Pathol Oral Radiol Endod 2011; 112:602-608.

15. Rovaldi CR, Pievsky A, Sole NA, Friden PM, Rothstein DM, Spacciapoli P. Photoactive porphyrin derivative with broad-spectrum activity against oral pathogens in vitro. Antimicrob Agents Chemother 2000;44:3364-3367.

16. Bagnato VS, Kurachi C. Controle microbiano com ação fotodinâmica: Uma revisão da presente tendência mundial. J Bras Laser 2010;2:8-14.

17. Fürst MM, Salvi GE, Lang NP, Persson GR. Bacterial colonization immediately after installation on oral titanium implants. Clin Oral Implants Res 2007;18:501-508.

18. Stranadko EP, Skobelkin OK, Mironov AE. Photodynamic therapy of cancer by photogem. In: Photodynamic Therapy of Cancer. G Jori, J Moan, WM Star (eds.). Proceedings of SPIE 2078, March 1, 1994; doi: 10.1117/12.168695.

19. Ahn J-C, Shin J-I, Chang S-Y, Kim H-J, Hong S-N. Antibacterial effect of photodynamic therapy using photogem and a $632 \mathrm{~nm}$ diode laser on Helicobacter pylori. J Exp Biomed Sci 2011;17:363-366.

20. Giusti JSM, Santos-Pinto L, Pizzolito AC, et al. Antimicrobial photodynamic action on dentin using a light-emitting diode light source. Photomed Laser Surg 2008;26:281-287.

21. Dovigo LN, Pavarina AC, de Oliveira Mima EG, Giampaolo ET, Vergani CE, Bagnato VS. Fungicidal effect of photodynamic therapy against fluconazole-resistant Candida albicans and Candida glabrata. Mycoses 2011;54:123-130.

22. Gois MM, Kurachi C, Santana EJB, et al. Susceptibility of Staphylococcus aureus to porphyrin-mediated photodynamic antimicrobial chemotherapy: an in vitro study. Lasers Med Sci 2010;25:391-395.

23. Winter S, Tortik N, Kubin A, Krammer B, Plaetzer K. Back to the roots: photodynamic inactivation of bacteria based on water-soluble curcumin bound to polyvinylpyrrolidone as a photosensitizer. Photochem Photobiol Sci 2013;12: 1795-1802.

24. Miquel J, Bernd A, Sempere J, Díaz-Alperi J, Ramírez A. The curcuma antioxidants: pharmacological effects and prospects for future clinical use. A review. Arch Gerontol Geriatr 2002;34:37-46.

25. Bruzell EM, Morisbak E, Tønnesen HH. Studies on curcumin and curcuminoids. XXIX. Photoinduced cytotoxicity of curcumin in selected aqueous preparations. Photochem Photobiol Sci 2005;4:523-530.

26. Epstein J, Sanderson IR, Macdonald TT. Curcumin as a therapeutic agent: the evidence from in vitro, animal and human studies. Br J Nutr 2010;103:1545-1557.

27. Haukvik T, Bruzell E, Kristensen S, Tønnesen HH. Photokilling of bacteria by curcumin in selected polyethylene glycol 400 (PEG 400) preparations: studies on curcumin and curcuminoids, XLI. Pharmazie 2010;65:600-606.

28. Ribeiro APD, Pavarina AC, Dovigo LN, et al. Phototoxic effect of curcumin on methicillin-resistant Staphylococcus aureus and L929 fibroblasts. Lasers Med Sci 2013;28: 391-398.

29. Dovigo LN, Pavarina AC, Carmello JC, Machado AL, Brunetti IL, Bagnato VS. Susceptibility of clinical isolates of Candida to photodynamic effects of curcumin. Lasers Surg Med 2011;43:927-934.

30. Kaur S, Modi NH, Panda D, Roy N. Probing the binding site of curcumin in Escherichia coli and Bacillus subtilis FtsZ - a structural insight to unveil antibacterial activity of curcumin. Eur J Med Chem 2010;45:4209-4214.

31. de Souza LM, Pratavieira S, Inada NM, Oliveira KT, Kurachi C, Bagnato VS. Evaluation of photodynamic effects of curcumin against the dengue vector-Aedes aegypti (Diptera: Culicidae). Photodiagnosis Photodyn Ther 2015;12:346-347.

32. Leite DPV, Paolillo FR, Parmesano TN, Fontana CR, Bagnato VS. Effects of Photodynamic therapy with blue light and curcumin as mouth rinse for oral disinfection: a randomized controlled trial. Photomed Laser Surg 2014;32: 627-632.

33. Araújo NC, Fontana CR, Gerbi MEM, Bagnato VS. Overall-mouth disinfection by photodynamic therapy using curcumin. Photomed Laser Surg 2012;30:96-101.

34. Dovigo LN, Carmello JC, de Souza Costa CA, et al. Curcumin-mediated photodynamic inactivation of Candida 
albicans in a murine model of oral candidiasis. Med Mycol 2013;51:243-251.

35. Ribeiro DG, Pavarina AC, Dovigo LN, et al. Photodynamic inactivation of microorganisms present on complete dentures. A clinical investigation Photodynamic disinfection of complete dentures. Lasers Med Sci 2012;27:161-168.

36. Enwemeka CS, Williams D, Enwemeka SK, Hollosi S, Yens D. Blue 470-nm light kills methicillin-resistant Staphylococcus aureus (MRSA) in vitro. Photomed Laser Surg 2009;27:221-226.

37. Kim S, Kim J, Lim W, et al. In vitro bactericidal effects of 625,525 , and $425 \mathrm{~nm}$ wavelength (red, green, and blue) light-emitting diode irradiation. Photomed Laser Surg 2013; 31:554-562.

38. Williams JA, Pearson GJ, Colles MJ, Wilson M. The effect of variable energy input from a novel light source on the photoactivated bactericidal action of toluidine blue o on Streptococcus mutans. Caries Res 2003;37:190-193.

39. Donnelly RF, McCarron PA, Tunney MM, David Woolfson A. Potential of photodynamic therapy in treatment of fungal infections of the mouth. Design and characterisation of a mucoadhesive patch containing toluidine blue O. J Photochem Photobiol B Biol 2007;86:59-69.
40. Pratavieira S, Agnelli PB, Dantas AAAR, Saad JRC, Bagnato VS, Rastelli ANS. Can different photosensitizers for oral photodynamic therapy provide color changes? In: IADR General Session 2012, Fós do Iguaçu, 2012.

41. Paschoal MA, Tonon CC, Spolidório DMP, Bagnato VS, Giusti JSM, Santos-Pinto L. Photodynamic potential of curcumin and blue LED against streptococcus mutans in a planktonic culture. Photodiagnosis Photodyn Ther 2013;10:313-319.

Address correspondence to: Sebastião Pratavieira São Carlos Institute of Physics University of São Paulo PO Box 369 São Carlos SP 13560-970 Brazil

E-mail: prata@ifsc.usp.br

Received: March 1, 2016. Accepted after revision: July 30, 2016. Published online: November 7, 2016. 